\title{
Ion-beam synthesis and thermal stability of highly tin-concentrated germanium - tin alloys
}

\section{Citation}

Tran, Tuan T., Hemi H. Gandhi, David Pastor, Michael J. Aziz, and J.S. Williams. 2017. “Ion-Beam Synthesis and Thermal Stability of Highly Tin-Concentrated Germanium - Tin Alloys." Materials Science in Semiconductor Processing 62 (May): 192-195. doi:10.1016/j.mssp.2016.10.049.

\section{Published Version}

doi:10.1016/j.mssp.2016.10.049

\section{Permanent link}

http://nrs.harvard.edu/urn-3:HUL.InstRepos:32094213

\section{Terms of Use}

This article was downloaded from Harvard University's DASH repository, and is made available under the terms and conditions applicable to Open Access Policy Articles, as set forth at http:// nrs.harvard.edu/urn-3:HUL.InstRepos:dash.current.terms-of-use\#OAP

\section{Share Your Story}

The Harvard community has made this article openly available.

Please share how this access benefits you. Submit a story.

Accessibility 


\section{Ion-beam synthesis and thermal stability of highly tin-concentrated germanium - tin alloys}

Tuan T. Tran ${ }^{1, a)}$, Hemi H. Gandhi ${ }^{2}$, David Pastor ${ }^{2}$, Michael J. Aziz ${ }^{2}$ and J. S. Williams ${ }^{1}$

${ }^{1}$ Department of Electronic Materials Engineering, Research School of Physics and Engineering, Australian National University, Canberra, ACT 0200, Australia

${ }^{2}$ Harvard John A. Paulson School of Engineering and Applied Sciences, Cambridge, Massachusetts, 02138, USA

A 9 at. \% Sn Ge-Sn alloy of good crystalline quality has been achieved by ion implantation followed by pulsed laser melting and resolidification. The concentration and crystallinity of the alloys are fully characterised by Rutherford backscattering spectrometry, X-ray diffraction, transmission electron microscopy and Raman spectroscopy. At high Sn concentrations, oxygen intermixing from a capping oxide layer, which is used to prevent ion-beam induced porosity, can interfere with the solidification process and compromise overall crystal quality. This indicates that the near surface layer containing oxygen after ion implantation must be removed before pulsed laser melting in order to obtain good crystal quality. The alloy's crystallinity is thermally stable under annealing up to $400^{\circ} \mathrm{C}$ for $30 \mathrm{~min}$. This thermal budget is comparable to that of Ge-Sn produced by conventional MBE or CVD methods and suitable for subsequent device fabrication and post-processing.

Over the last few years the semiconductor research community has directed considerable effort towards developing group IV germanium-tin (Ge-Sn) binary alloys. This interest in the Ge-Sn material platform stem from its attractive high performance optoelectronic properties and its potential deployment to solve long-anticipated problems in front-end-of-line (FEOL) and backend-of-line (BEOL) electronic device processing. Ge-Sn alloy (11 at.\%Sn) has been calculated to have four times higher carrier mobility compared to non-alloyed Ge, indicating high suitability for FEOL transistors ${ }^{1}$. For newly proposed tunnel field effect transistors, use of fundamental direct bandgap semiconductor like the Ge-Sn alloy could enhance tunnelling probability and related drive-current, allowing operation of devices beyond theoretical subthreshold slope limitations of $60 \mathrm{mV} / \mathrm{dec}^{2,3}$. For BEOL structure and data communication, the prospect of transmitting signals using photons is tantalising for ultimate speed and low power consumption. To facilitate this, there has been much effort in integrating photonic materials, like direct bandgap III-V semiconductors, into Si CMOS circuitry. For example, the first end-to-end silicon photonics integrated link operated at $50 \mathrm{Gbit} \mathrm{s}^{-1}$ over a 
single fibre has been demonstrated by Intel's Photonics Technology Labs ${ }^{4}$. While this result was promising, it relied on cumbersome III-V bonding of an indium phosphide layer to a silicon photonic chip. An alternative, potentially lower cost solution would be to use Si CMOS compatible direct bandgap semiconductor like Ge-Sn alloys for light emission. A proof-ofconcept optically pumped laser from a Ge-Sn alloy has been demonstrated, where the material was grown directly on a silicon substrate by a chemical vapour deposition technique (CVD) ${ }^{5}$.

Recently, Ge-Sn alloys have also been produced by ion beam synthesis using ion implantation and nanosecond pulsed laser melting (PLM) ${ }^{6,7}$. This fabrication method is potentially advantageous over standard Ge-Sn fabrication via CVD in that it may allow for higher Sn fraction, easier post fabrication pathway for layer lattice relaxation and device implementation. A single crystalline Ge-Sn alloy with $\sim 6 a t$. \% Sn has been achieved using this method, with excellent crystal quality except for some local pore formation that occurs after ion implantation ${ }^{7}$. These defective regions have been shown to be a consequence of ion-beam induced porosity in Ge, which also increases the loss of Sn during implantation ${ }^{8}$. By using a nanometer scale pre-implantation capping layer of silicon dioxide $\left(\mathrm{SiO}_{2}\right)$, the porosity and $\mathrm{Sn}$ loss due to sputtering are both significantly suppressed as shown in Ref. ${ }^{8}$. After implantation, a Sn content of $\sim 15 \mathrm{at}$. \% has been achieved for a Ge substrate with a $40 \mathrm{~nm}$ capping layer. No sign of surface porosity can be detected in these samples.

Developed from the work in Ref. ${ }^{8}$ which focused mostly on the suppressing effect of the capping layer, this work presents the fabrication aspect of the Ge-Sn alloys with detailed physical characterisation including Rutherford backscattering spectrometry (RBS), X-ray diffraction (XRD), transmission electron microscopy (TEM) and Raman spectroscopy. Furthermore, the thermal stability of the alloys, which is crucial information for further application of the materials, will also be presented in this contribution.

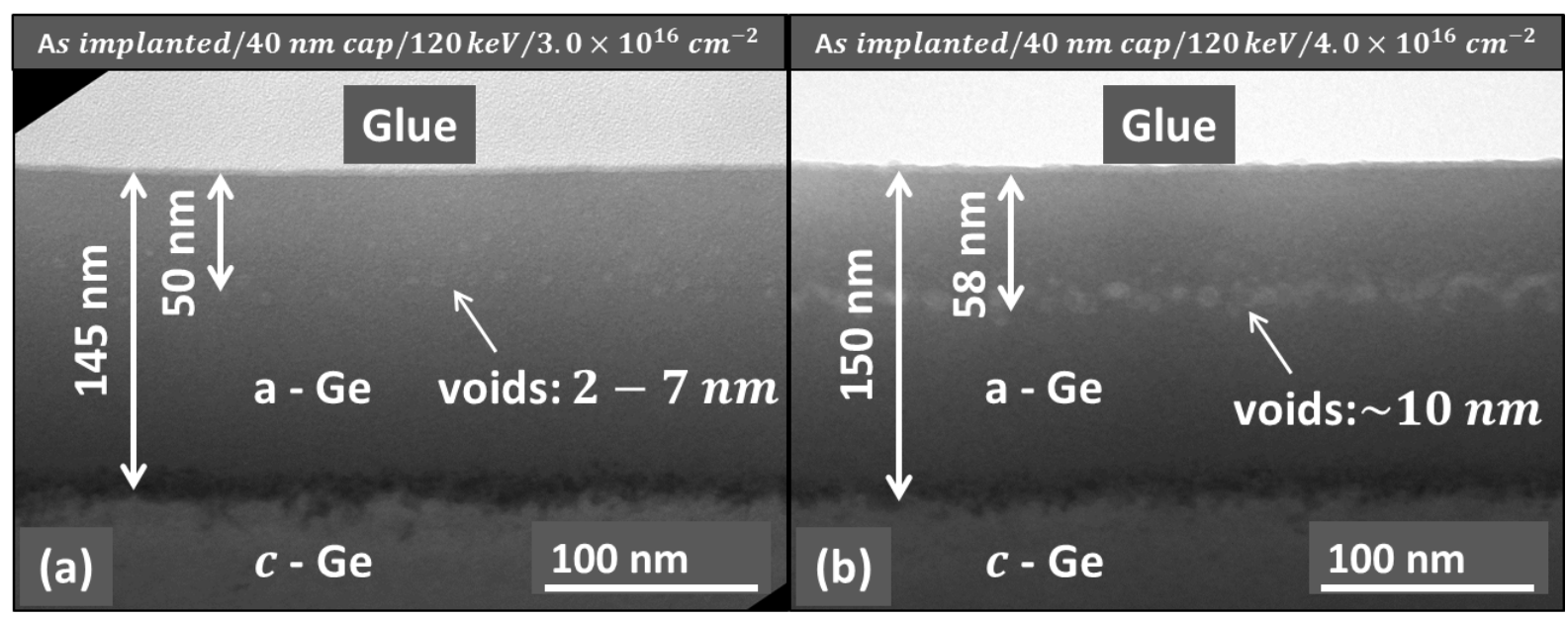


Fig. 1: TEM micrographs of the as-implanted samples at the dose of $3.0 \times 10^{16} \mathrm{~cm}^{-2}$ (Fig. 1(a)) and $4.0 \times 10^{16} \mathrm{~cm}^{-2}$ (Fig. 1(b)). In Fig. 1(a), a band of nanometer scale voids occurred at the depth of the maximum vacancy production. This band grows larger with higher implant dose in Fig. 1(b).

Prior to ion implantation, several p-type (Ga doped) (100) Ge substrates were coated with a $\sim 40 \mathrm{~nm} \mathrm{SiO} 2$ by plasma enhanced chemical vapour deposition. The capped substrates were implanted with ${ }^{120} \mathrm{Sn}^{-}$at an energy of $120 \mathrm{keV}$ and the implant doses were in the range of $2.5-$ $4.5 \times 10^{16} \mathrm{~cm}^{-2}$. All the substrates were misoriented $7^{\circ}$ to the [100] crystal axis of the substrate to minimise channelling, and also were kept at liquid nitrogen temperature $\left(\mathrm{LN}_{2} \mathrm{~T}\right)$ to suppress ion-beam-induced porosity ${ }^{9,10}$.

Fig. 1 shows the TEM micrographs of the Ge substrates after the implantation of Sn and after the removal of the capping oxide layer by hydrofluoric acid (HF). The effectiveness of the capping layer in preventing porosity is clearly shown in Fig. 1(a) and 1(b), where the surface remains atomically flat surface. However, it is worth noting that at the depth of $50 \mathrm{~nm}$ below the surface there is a band of voids measured from $2 \mathrm{~nm}$ to $7 \mathrm{~nm}$ in the $3.0 \times 10^{16} \mathrm{~cm}^{-2}$ sample (Fig. 1(a)). Beyond the dose of $3.0 \times 10^{16} \mathrm{~cm}^{-2}$, the size of the voids increases to $>$ $10 \mathrm{~nm}$ at a higher implant dose of $4.0-4.5 \times 10^{16} \mathrm{~cm}^{-2}$ (Fig. 1(b)). Further investigation will be presented later to clarify whether this band of voids can be annihilated by a PLM process. Subsequently, PLM was achieved using a frequency tripled Nd:YAG laser (355 $\mathrm{nm}, 4 \mathrm{~ns}$ ) with a single pulse of $\sim 0.4 \mathrm{~J} \mathrm{~cm}^{-2}$. During the process, time resolved reflectivity measurements performed with an Ar ion $(488 \mathrm{~nm})$ laser source provided a melt duration of $53-66 n s$. Crystal quality of the samples are characterised by Rutherford backscattering spectrometry (RBS), X-ray diffraction (XRD), Raman spectroscopy and transmission electron microscopy (TEM).
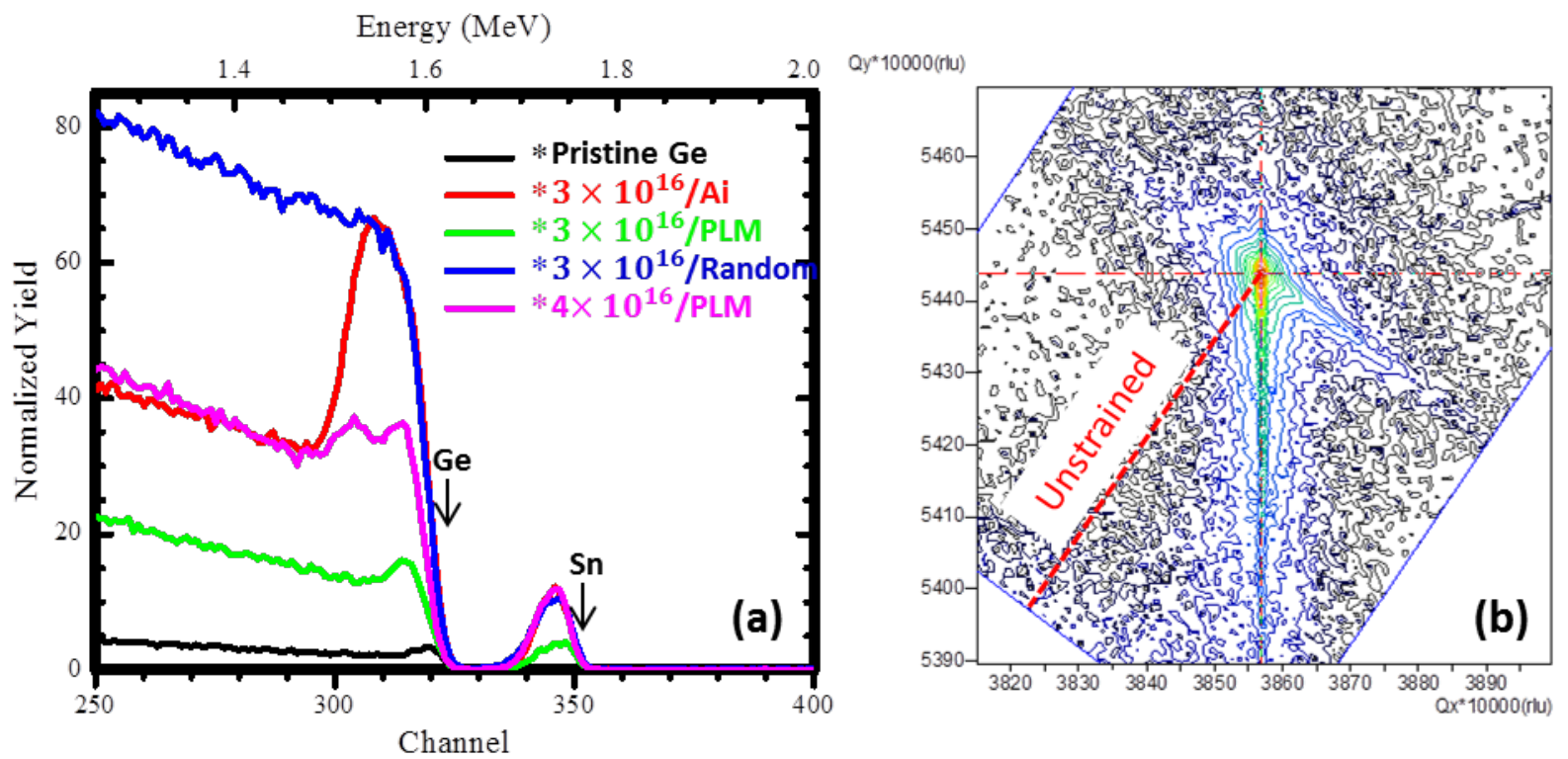
Fig. 2: Channelled RBS spectra of the $3.0 \times 10^{16} \mathrm{~cm}^{-2}$ sample show that about $85-90 \%$ of Sn is substitutional (Fig. 2(a)). In Fig. 2(b), XRD/reciprocal space mapping shows the crystalline Ge-Sn alloy is compressively strained in the (100) planes.

After PLM, RBS spectra were taken on the $3.0 \times 10^{16} \mathrm{~cm}^{-2}$ and $4.0 \times 10^{16} \mathrm{~cm}^{-2}$ samples in channelling and random configurations to characterise the physical properties of the samples after PLM. The probing beam of the RBS system is ${ }^{4} \mathrm{He}^{+}$at an energy of $2 \mathrm{MeV}$. The Sn concentration of the $3.0 \times 10^{16} \mathrm{~cm}^{-2}$ sample after implantation is $\sim 9.5 \mathrm{at}$. \% as determined by fitting the red spectrum in Fig. 2(a) with a simulated RUMP spectrum ${ }^{11}$. The Sn profile of the sample is Gaussian-like as expected for a non-porous Ge sample. After PLM, the crystal structure of the Ge-Sn layer recovered very well as indicated by the reduced scattering yield of the green spectrum. By comparing random and channelled RBS spectra, the substitutionality of $\mathrm{Sn}$ atoms is calculated to be $85-90 \%$. Although the Sn profile has been redistributed slightly during PLM, the peak concentration of Sn in Ge is estimated to be $\sim 9$ at. \%. This result is certainly encouraging as it is comparable to some of the best studies using molecular beam epitaxy ${ }^{12,13}$ and chemical vapour deposition ${ }^{14,15}$. For the $4.0 \times 10^{16} \mathrm{~cm}^{-2}$ sample, the high scattering yield of the channelling spectrum (purple) represents a disordered layer, noting the two large bumps: one at the sample's surface and one at about the back edge of the amorphous layer. These two features will be clarified in the following TEM figures.

Fig. 2(b) is an X-ray reciprocal space map of the $3.0 \times 10^{16} \mathrm{~cm}^{-2}$ sample after PLM. The map was constructed by doing a series of $\omega-2 \theta$ scans at slightly different $\omega$ offsets around the asymmetric reciprocal point $(\overline{2} \overline{2} 4)$. This asymmetrical mapping is able to show the in-plane $\left(Q_{x}\right)$ as well as the out-of-plane lattice expansion $\left(Q_{y}\right)$, so that any strain relaxation can be observed. At the cross of the figure is the reciprocal point $(\overline{2} \overline{2} 4)$ from the substrate. The perfectly vertical streak going downwards from the substrate's point is from the compressively strained Ge-Sn layer. This result of strain is usually expected for good epitaxy of a thin layer in which there is uniaxial strain perpendicular to the film as a result of the film accommodating the same lateral lattice parameter as the substrate. However, studies have shown that the biaxial compressive strain as in this case has a negative impact in the Ge-Sn layer in terms of the direct bandgap transition as it requires a higher concentration of Sn to compensate for the strain ${ }^{16}$. 


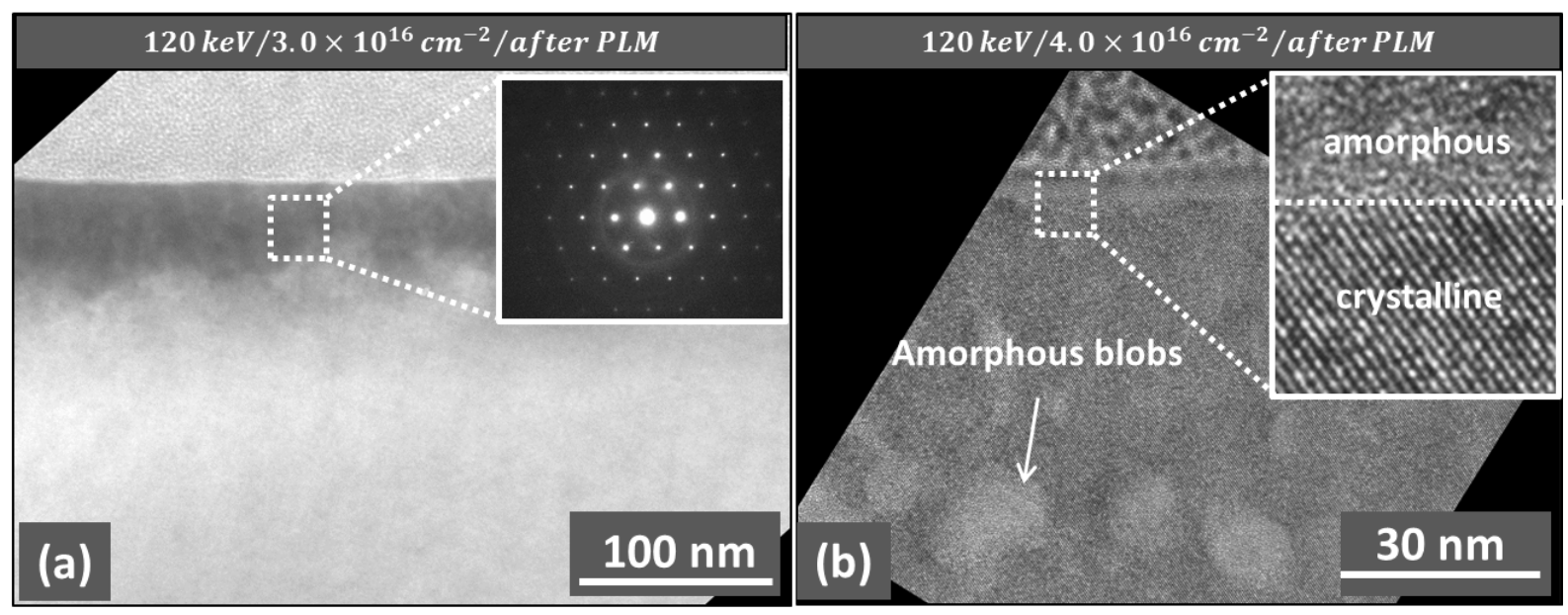

Fig. 3: TEM micrographs of the capped samples (Fig. 3(a) and 3(b)) after PLM. Electron diffraction pattern (inset, Fig. 3(a)) of the $3.0 \times 10^{16} \mathrm{~cm}^{-2}$ sample shows a typical diamond-cubic monocrystal. In Fig. 3(b), a band of disordered, amorphous blobs can be observed in the $4.0 \times 10^{16} \mathrm{~cm}^{-2}$ sample. The high resolution TEM (inset, Fig. 3(b)) shows good crystal quality around the disordered blobs.

Further TEM analysis was again undertaken to characterise the crystallinity of the Ge-Sn layer after PLM. Fig. 3(a) shows that the small band of $2-7 \mathrm{~nm}$ voids in Fig. 1(a) can be annihilated by the PLM process for the $3.0 \times 10^{16} \mathrm{~cm}^{-2}$ case. No significant disorder and extended defects have been observed in this sample. Electron diffraction conducted within the Ge-Sn layer of this sample (inset) shows a typical pattern of a diamond cubic structure. The blurry ring within the pattern was identified to be from an amorphous platinum layer deposited on the Ge samples during focused-ion-beam sample preparation. However, for the higher dose case in Fig. 1(b) it is shown in Fig. 3(b) that the voids and impurities following implantation give rise to residual disorder after PLM in the form of large amorphous regions. Our energy-dispersive X-ray spectroscopy data show that the relative concentration of Sn changes only slightly within these regions; i.e. these blobs are not Sn precipitates. Meanwhile, the concentration of oxygen (O) significantly increases as indicated by an EDX scan across the blobs. Therefore, it is hypothesised that $\mathrm{O}$ atoms in the capping layer are intermixed with the substrate during the implantation. Although the intermixing layer is shallow, within $5 \mathrm{~nm}$ of the surface, $\mathrm{O}$ appears to be relocated towards the voids during the melting stage, thus hindering a full recovery of the lattice. Similarly, the intermixed oxygen appears to retard the recrystallization at the sample's surface as shown in the upper part of the Fig. 3(b)'s inset. The amorphous regions at the surface and close to the back edge of the Ge-Sn layer are consistent with the two large bumps in the RBS spectrum mentioned previously. It is therefore necessary to remove at least the first $5 \mathrm{~nm}$ of the sample's surface prior to PLM by a controlled etching process such as reactive ion etching to remove the intermixed $\mathrm{O}$ and achieve a better quality crystalline alloy layer. 


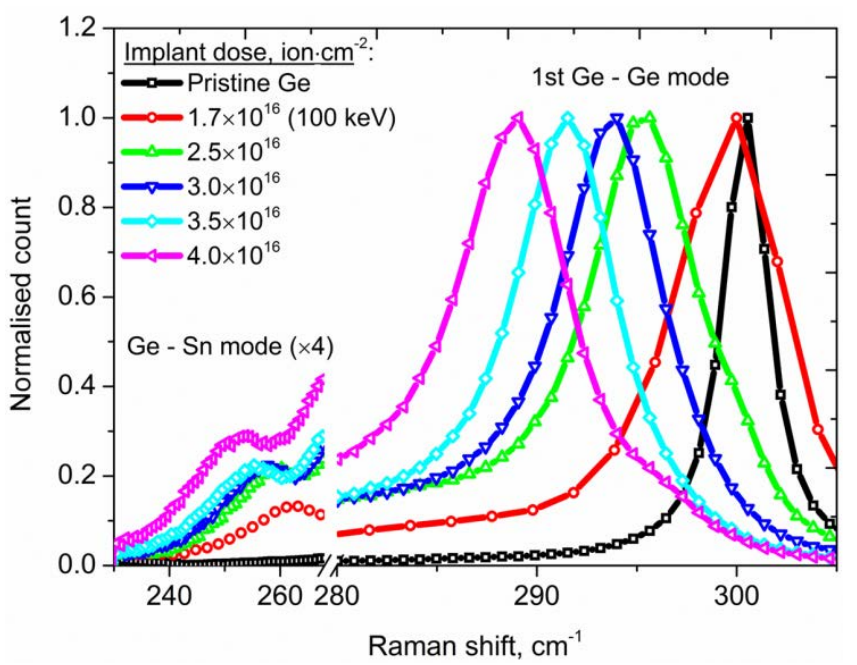

Fig. 4: Raman spectra of the Ge-Sn samples after PLM. The intensity of the Ge-Sn phonon mode consistently increases with higher Sn implant doses of Sn. As a result of the increasing substitutional Sn, the $1^{\text {st }}$ order Ge-Ge phonon mode shifts towards lower Raman shift.

Raman spectroscopy of post-PLM samples are conducted to study bonding arrangements within the Ge-Sn lattice, most noticeably the Ge-Sn local vibration phonon mode and the $1^{\text {st }}$ order Ge-Ge phonon mode. This Raman spectroscopy was performed using a Renishaw 2000 micro-Raman instrument with a red laser source (He:Ne, $633 \mathrm{~nm}$ ) and a $2400 \mathrm{l} / \mathrm{mm}$ grating giving a spectra resolution of $\sim 0.8$ wavenumber (Raman shift). Since most of the laser power is absorbed within $\sim 40 \mathrm{~nm}$ of the surface ${ }^{17}$, only the Raman data from the Ge-Sn layer is examined without contribution from the underlying Ge substrate. For the pristine Ge sample (square-black), the $1^{\text {st }} \mathrm{Ge}-\mathrm{Ge}$ mode is located at a wavenumber of $300.6 \mathrm{~cm}^{-1}$ and there is no sign of the Ge-Sn mode. With the introduction of Sn into the Ge lattice, an additional peak arises at a wavenumber of $259.4 \mathrm{~cm}^{-1}\left(2.5 \times 10^{16} \mathrm{~cm}^{-2}\right.$ sample, green-triangle), which represents the substitutionality of $\mathrm{Sn}$ in Ge lattice ${ }^{18}$. The intensity of the Ge-Sn phonon mode monotonically increases with the Sn concentration. As a consequence of the substitutional Sn atoms and the uniaxial strain, the $1^{\text {st }} \mathrm{Ge}$-Ge mode consistently shifts to a lower wavenumber. The wavenumber difference between the pristine Ge and the highest dose sample is $11.6 \mathrm{~cm}^{-1}$. It is widely known that the shift of the $1^{\text {st }} \mathrm{Ge}-\mathrm{Ge}$ phonon mode is a function of both the concentration of substitutional Sn and the in-plane strain of the Ge-Sn lattice: $\Delta \omega_{G e-S n}=$ $\omega_{G e}-\omega_{G e-S n}=a \times C_{S n}+b \times \varepsilon_{\text {in-plane }}$, where $\mathrm{a}$ and $\mathrm{b}$ are constants. According to Ref. ${ }^{19}$, for a fully strained Ge-Sn alloy, the shift equation can be simplified to $\Delta \omega_{G e-S n}=76.8 \times C_{S n}$. The Sn concentration of the $3.0 \times 10^{16} \mathrm{~cm}^{-2}$ sample is $\sim 9$ at. $\%$ and if we assume that the $\mathrm{X}$ ray diffraction analysis indicates a fully strained Ge-Sn material (Fig. 2(b)), at this concentration the calculated shift of the $1^{\text {st }} \mathrm{Ge}-\mathrm{Ge}$ peak is $6.9 \mathrm{~cm}^{-1}$. Our experimental data for this sample indicates a shift of $6.5 \mathrm{~cm}^{-1}$, in good agreement with the calculated value, 
indicating a fully strained Ge-Sn layer. For the $4.0 \times 10^{16} \mathrm{~cm}^{-2}$ sample, the calculated and the experimental data are $\sim 9.2 \mathrm{~cm}^{-1}$ and $11.6 \mathrm{~cm}^{-1}$ respectively. The large discrepancy between the two values is probably due to a partial relaxation of the Ge-Sn lattice through the introduction of defects, such as the disordered blobs, at such a high Sn concentration. Due to this relaxation, the second term of the shift expression becomes larger, giving rise to a larger shift as compared to a fully strained layer.

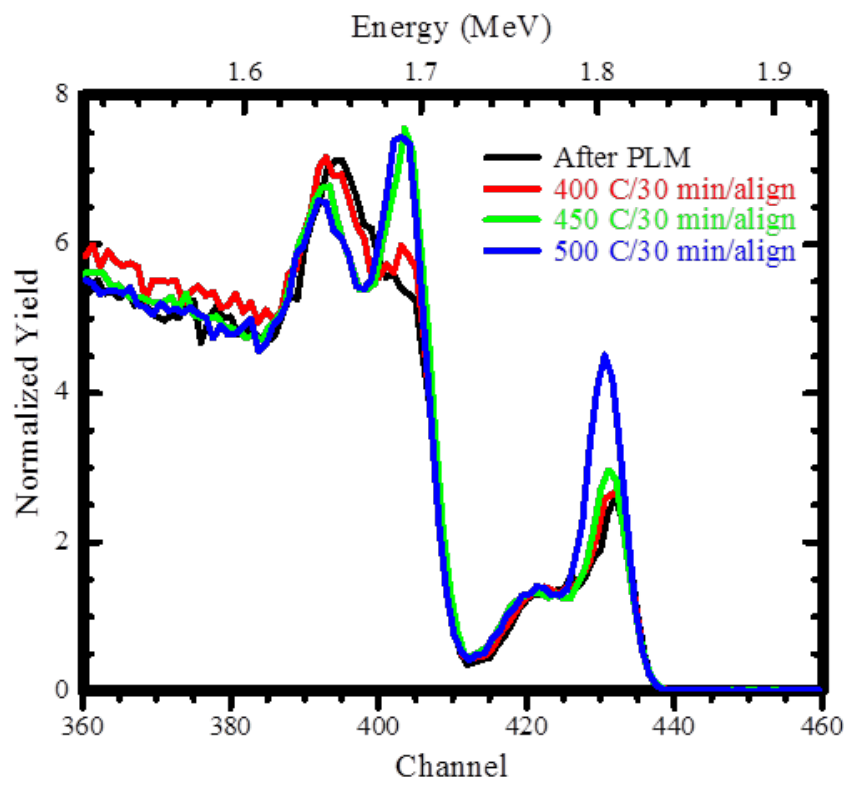

Fig. 5: RBS/channelling spectra of $3.0 \times 10^{16} \mathrm{~cm}^{-2}$ PLMed samples before and after annealing at $400^{\circ} \mathrm{C}, 450^{\circ} \mathrm{C}$ and $500^{\circ} \mathrm{C}$ for $30 \mathrm{~min}$. There is no observable difference between the RBS spectra of the $400^{\circ} \mathrm{C}$ annealed and non-annealed sample, indicating no change in crystal quality. The spectra of the $450^{\circ} \mathrm{C}$ and $500^{\circ} \mathrm{C}$ annealed samples show a Sn signal at the surface that increases with anneal temperature, indicating surface segregation.

Finally, the PLMed $3.0 \times 10^{16} \mathrm{~cm}^{-2}$ sample with good crystal quality was annealed at various temperatures to study its thermal stability and suitability for device processing. The range of annealing temperatures varied from $250^{\circ} \mathrm{C}$ to $500^{\circ} \mathrm{C}$ in increment of $25^{\circ} \mathrm{C}$. Each anneal was conducted over the course of $30 \mathrm{~min}$ in an argon atmosphere with a flow rate of $100 \mathrm{sccm}$. Each annealed sample was characterised with the RBS/channelling technique to determine the diffusion and substitutionality of Sn atoms. The RBS/channelling spectra of the $3.0 \times$ $10^{16} \mathrm{~cm}^{-2}$ sample are largely unchanged from $250^{\circ} \mathrm{C}$ to $400^{\circ} \mathrm{C}$ relative to the non-annealed spectra. As seen in Fig. 5, the Sn profile begins to change after an anneal temperature of $\sim 400^{\circ} \mathrm{C}$. The degree of Sn segregation to the surface increases with increasing temperature between $450^{\circ} \mathrm{C}$ and $500^{\circ} \mathrm{C}$. In addition, comparing the channelled spectra in Fig. 5 with corresponding random spectra shows that a corresponding loss of Sn substitutionality occurs beyond $400^{\circ} \mathrm{C}$ annealing. In summary, the crystalline Ge-Sn alloys are stable under annealing up to $400^{\circ} \mathrm{C}$ for $30 \mathrm{~min}$. This result is in good agreement with other reports ${ }^{20,21}$ for alloys prepared by CVD and MBE methods. 
In conclusion, the data in this paper shows the method of ion implantation followed by pulse laser melting is capable of producing a Ge-Sn alloy with a Sn concentration of $\sim 9$ at.\%. Detailed characterisation using RBS, XRD and TEM demonstrate the good crystallinity of the $3.0 \times 10^{16} \mathrm{~cm}^{-2}$ sample as the nanometer scale voids can be annihilated during the laser melting process. However, for $4.0 \times 10^{16} \mathrm{~cm}^{-2}$ sample larger voids and more extensive oxygen intermixing from the capping layer interferes with good epitaxial growth during resolidification. Oxygen intermixing also appears to inhibit the crystallisation at the surface. This data suggest a pre-etching step would be necessary to remove the intermixing layer and improve the sample quality. The thermal stability study shows that the highly Sn concentrated alloy is quite stable as it can sustain under thermal annealing up to $400^{\circ} \mathrm{C}$ for $30 \mathrm{~min}$. This thermal budget makes the material highly applicable for manufacturing electronic and photonic devices.

\section{ACKNOWLEDGEMENT}

The authors would like to acknowledge the Australian Research Council for the funding support, the National Collaborative Research Infrastructure Strategy for the access to the Australian National Fabrication Facility and the Heavy Ion Accelerator Facility. D.P. acknowledges financial support from the MEC within Programa Nacional de movilidad de recursos humanos del Plan Nacional I+D+i 2008-2011 (EX-2010-0662) and to the program Ramón y Cajal (RYC-2014-16936). This work was also supported by the US Air Force Office of Scientific Research (FA9550-14-1-0150). 
$1 \quad$ J. D. Sau and M. L. Cohen, Physical Review B 75, 045208 (2007).

2 R. Kotlyar, U. E. Avci, S. Cea, R. Rios, T. D. Linton, K. J. Kuhn, and I. A. Young, Applied Physics Letters 102, 113106 (2013). K. H. Kao, A. S. Verhulst, W. G. Vandenberghe, B. Soree, G. Groeseneken, and K. D. Meyer, IEEE Transactions on Electron Devices 59, 292 (2012).

Nat Photon 4, 498 (2010).

S. Wirths, R. Geiger, N. v. d. Driesch, G. Mussler, T. Stoica, S. Mantl, Z. Ikonic, M. Luysberg, S. Chiussi, J. M. Hartmann, H. Sigg, J. Faist, D. Buca, and D. Grützmacher, Nat Photon 9, 88 (2015). K. Gao, S. Prucnal, R. Huebner, C. Baehtz, I. Skorupa, Y. Wang, W. Skorupa, M. Helm, and S. Zhou, Applied Physics Letters 105, 042107 (2014). T. T. Tran, D. Pastor, H. H. Gandhi, L. A. Smillie, A. J. Akey, M. J. Aziz, and J. S. Williams, Journal of Applied Physics 119, 183102 (2016). T. T. Tran, H. S. Alkhaldi, H. H. Gandhi, D. Pastor, L. Q. Huston, J. Wong-Leung, M. J. Aziz, and J. S. Williams, Applied Physics Letters 109, 082106 (2016).

O. W. Holland, B. R. Appleton, and J. Narayan, Journal of Applied Physics 54, 2295 (1983).

B. Stritzker, R. G. Elliman, and J. Zou, Nuclear Instruments and Methods in Physics Research Section B: Beam Interactions with Materials and Atoms 175-177, 193 (2001). L. Doolittle and M. Thompson, (1990).

R. Chen, H. Lin, Y. Huo, C. Hitzman, T. I. Kamins, and J. S. Harris, Applied Physics Letters 99 (2011). Y. Shimura, N. Tsutsui, O. Nakatsuka, A. Sakai, and S. Zaima, Thin Solid Films 518, S2 (2010). Domulevicz, A. Nazzal, G. Sun, R. A. Soref, J. Tolle, B. Li, H. A. Naseem, and S.-Q. Yu, Applied Physics Letters 105 (2014).

C. L. Senaratne, J. D. Gallagher, L. Jiang, T. Aoki, D. J. Smith, J. Menéndez, and J. Kouvetakis, Journal of Applied Physics 116, 133509 (2014).

S. Gupta, B. Magyari-Köpe, Y. Nishi, and K. C. Saraswat, Journal of Applied Physics 113, 073707 (2013).

M. Brown and C. Arnold, in Laser Precision Microfabrication; Vol. 135, edited by K. Sugioka, M. Meunier, and A. Piqué (Springer Berlin Heidelberg, 2010), p. 91. H. Lin, R. Chen, Y. Huo, T. I. Kamins, and J. S. Harris, Applied Physics Letters 98, 261917 (2011).

M. Rojas-López, H. Navarro-Contreras, P. Desjardins, O. Gurdal, N. Taylor, J. R. A. Carlsson, and J. E. Greene, Journal of Applied Physics 84, 2219 (1998).

R. Chen, Y.-C. Huang, S. Gupta, A. C. Lin, E. Sanchez, Y. Kim, K. C. Saraswat, T. I. Kamins, and J. S. Harris, Journal of Crystal Growth 365, 29 (2013).

H. Li, Y. X. Cui, K. Y. Wu, W. K. Tseng, H. H. Cheng, and H. Chen, Applied Physics Letters 102, 251907 (2013). 